\title{
Gracias Instituto Asenjo
}

\author{
Luis Carlos Cadavid ${ }^{1}$ \\ 1 Neurocirujano Estereotáctico - Hospital Universitario San Vicente de Paul - U de Antioquía, Medellín. Colombia.
}

Rev. Chil. Neurocirugía 42: 71-75, 2016

\begin{abstract}
Resumen
El objeto de este artículo es que agradecer a la Sociedad Neurológica de Chile la influencia que ha tenido en Antioquia y Colombia formando nuestros lideres en neurocirugía, especialmente por haber formado al Doctor Ernesto Bustamante, fundador del Servicio de Neurocirugía de la Universidad de Antioquia que cumple 65 años de existencia. Sin embargo, este no fue el único Neurocirujano de Colombia que recibió su entrenamiento en Chile, también lo fueron los Doctores Ernesto Arango Tamayo, Alejandro Jiménez Arango, Raúl Piedrahita, Carlos Pardo, Bernardo Soto, Hans Carmona, Alveiro Morales y muchos otros cuyo nombre desconozco. Sea esta la oportunidad para retomar y fortalecer los lazos académicos entre nuestras sociedades científicas.

Gracias Instituto Asenjo
\end{abstract}

Palabras clave: Historia, Instituto Asenjo, Neurocirugía Colombia, Ernesto Bustamente Zuleta.

\begin{abstract}
The main purpose of this article is to recognize the great influence of the Chilean neurosurgical society in the history and development of Colombian neurosurgery. Two pioneers in Colombian neurosurgery: Dr. Alejandro Arango and Ernesto Bustamante were trained by Dr. Alfonso Asenjo in earlier years of the Institute. Dr. Ernesto Bustamante was the founder of the First Neurosurgical and Neurogical service in Colombia, at the Hospital Universitario San Vicente de Paul (Universidad de Antioquia) that is now celebrating 66 years of existence with more tan 50 Neurologist and 70 neurosurgeons formed. The influence of the Chilean neurosurgery has continued since then and many neurosurgeons had visited Chile in order to improve their knowledge. This is an oportunity to strenght the academic link between our cientific societies. Thanks Instituto Asenjo.
\end{abstract}

Key words: History, Instituto Asenjo, Colombian Neurosurgery, Ernesto Bustamante Zuleta.

La historia de la Neurocirugía de Antioquia tiene su origen más remoto en 1893 cuando el Doctor Tomas Quevedo hizo la cirugía de un tumor cerebral que el mismo diagnosticó ${ }^{1}$, esta cirugía tiene un valor histórico similar a la realizada 9 años antes por Godlee y Bennett en $1884^{2}$.

El 7 de noviembre de 1950 el Doctor Ernesto Bustamante dio inicio al Servicio de Neurología y Neurocirugía del Hospital Universitario San Vicente de Paul la historia clínica número uno, el primer paciente fue el señor José Nolasco Morales a quien se le practico una cirugía de un tumor medular, después de esto han sido miles los pacientes atendidos por neurólogos y neurocirujanos formados en este centro académico; el servicio ha estado en actividad continua desde esa época, constituyéndose en el más antiguo de Colombia.

Por ser el servicio que conserva las historia clínicas desde su fundación se puede afirmar que aquí reposa la historia de la Neurología y Neurocirugía de

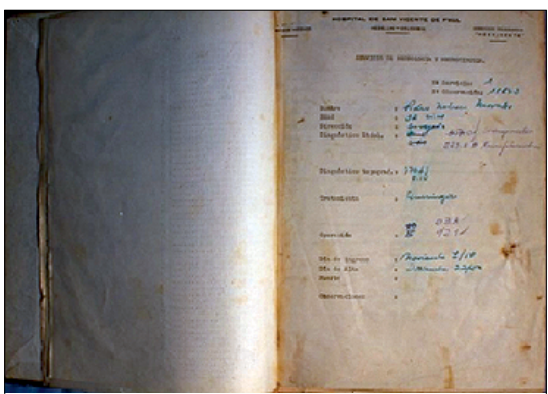

Figura 1. Historia Clínica número 1 , noviembre 7 de 1950. 
Colombia, los primeros diagnósticos y también las primeras cirugías se hicieron en este servicio mucho antes que en otras ciudades como se puede demostrar por el archivo clínico.

La fundación del servicio fue precedido por el interés por las neurociencias en los años 40 del siglo pasado; en 1942 regreso a Medellín el Dr. Ernesto Arango Tamayo, se gradúo en la Universidad de Antioquia como Médico Cirujano y viajo a Europa a perfeccionar sus conocimientos, cuentan que a su regreso el barco atraco en Santiago de Chile, visito el Servicio del Dr. Asenjo que estaba en el período de gestación y se quedó allí como "ayudante extranjero", a su regreso Medellín publicó un artículo en el que describía su experiencia en la cirugía de "meningiomas"; reconoció la necesidad de un entrenamiento y un grupo quirúrgico especializados, afirmó: "No debe nunca intentarse una intervención sobre la masa encefálica sin un equipo quirúrgico especializado y de un sincronismo en el trabajo perfecto; ya sabemos los tristes resultados obtenidos en manos de cirujanos generales en esta clase de operaciones; no por ser buen cirujano de las vías gástricas se puede invadir el campo de la Neurocirugía"3; en la ciudad se dedicó a la especialidad de "Órganos de los sentidos" pero su trabajo como neurocirujano fue muy poco y no dejó ninguna huella, es más recordado por su personalidad excéntrica.

El Doctor Bustamante reconoce que fue un alumno de Ramón y Cajal, el Doctor Antonio Pedro Rodríguez Pérez, quien lo motivó, a él y muchos otros, en el estudio de neurociencias. Este médico español, oficial del ejército republicano, al terminar la guerra civil española, emigró a Venezuela inicialmente y luego a Colombia, fue Jefe de Morfología en la Universidad de Antioquia.

En las primeras publicaciones sobre la Neurocirugía se puede ver que existió interés por desarrollar los métodos de diagnostico neuroradiologico especialmente, son muy pocas las publicaciones sobre los aspectos clínicos, en mi caso más me he interesado en conocer el desarrollo de la Neurocirugía.

En las promociones medicas de los años 46 y 47 del siglo pasado varios médicos sintieron interés por la Neurocirugía, uno de ellos, el Doctor Luis Carlos Posada viajó a especializarse en Neurocirugía en Ann Arbor, Michigan, U.S.A, los doctores Ernesto
Bustamante y Alberto Sion publicaron sus tesis de grado sobre la yodoventriculogarfía y arteriografía, las técnicas de neurodiagnóstico más importantes hasta la aparición de la Tomografía, fueron los pioneros de la neuroradiología en Colombia.

Siendo estudiante el Doctor Ernesto Bustamante Zuleta se distinguió entre sus compañeros por su orientación neuroquirúrgica, sus profesores compartían con el casos clínicos y lo invitaban a participar en las cirugías como ayudante aún antes de graduarse, uno de sus profesores, director del servicio de urgencias, el Doctor Joaquín Aristizabal lo autorizaba para atender y operar pacientes urgentes en el Servicio de Policlínica. El primer caso de Espongioblasoma Multiforme Precoronal fue publicado en 1946, se presenta el caso clínico y se hace un detallado análisis neuroanatómico y se correlaciona con los hallazgos quirúrgicos. Durante la cirugía se inyectó Lipiodol en el quiste y se tomaron placas radiográficas para conocer su tamaño y localización; en la cirugía participo el Doctor Bustamante y seguramente él fue quien tuvo la idea de inyectar el medio de contraste ${ }^{4}$, en el mismo año publicó el primer tumor de la Pineal en Colombia, tumor diagnosticado por la Yodoventriculografia $^{5}$ y en 1947 presentó su tesis de grado mostrando su experiencia en 13 casos $^{6}$, esta tesis es de alta calidad científica, en ella se aprecia la correcta interpretación de las imágenes.

Otro médico que se intereso en las técnicas de neurodiagnóstico fue el $\mathrm{Dr}$. Alberto Sion, hizo su tesis sobre "Arteriografías periféricas y angiografías cerebrales", estos procedimientos eran bastante difíciles de realizar por la falta de conocimiento, no se habían hecho antes en Colombia; además se carecía de los equipos adecuados.

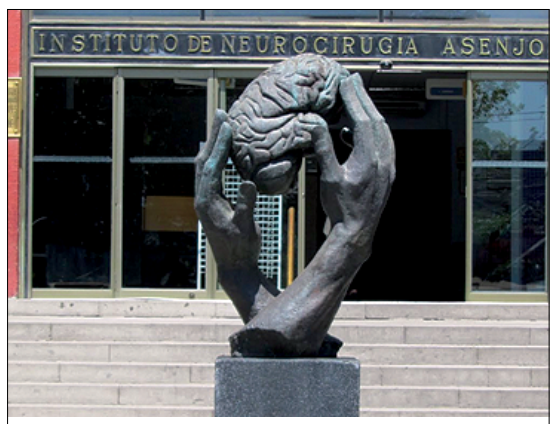

Figura 2. Escultura "Homenaje a la Neurocirugía" Obra de Marta Colvin.
Fue especialmente difícil hacer las primeras arteriografías cerebrales, la punción directa de la carótida era difícil porque no se disponía de las agujas para arteriografía y a veces era necesaria la disección de la arteria, uso dos medios de contraste; el Yoduro de sodio lo descarto porque no daba buen contraste radiológico, el Neiopax tenía la desventaja de producir mucho dolor y necesitaba aplicar $0,5 \mathrm{gr}$ de Pentotal como analgésico, la hipotensión y convulsiones también fueron frecuentes, aparte de las dificultades técnicas la disponibilidad del equipo de radiología era muy restringida y la colaboración del personal paramédico fue muy poca, planeo hacer 100 arteriografías pero sólo pudo hacer 13 y solamente describe dos arteriografías cerebrales, en una hizo el diagnóstico de arteritis senil y en otra aneurisma de carótida ${ }^{7}$.

Continuando con la introducción de los métodos diagnósticos en el país encontramos que el Pantopaque fue descubierto en 1942 y 2 años más tarde el Doctor Oriol Arango lo empleo en la ciudad y publico sus resultados en $1947^{\circ}$. El doctor Gabriel llano hizo su tesis de grado sobre la mielografia, describe el cuadro clínico de la hernia del disco y los hallazgos radiológicos, en su tesis reporta la primera cirugía de hernia de disco de la ciudad ya que los pacientes con hernia de disco eran remitidos para tratamiento a Estados Unidos ${ }^{9}$.

El Doctor Bustamante tuvo conocimiento del Servicio del Doctor Asenjo por el Doctor Ernesto Arango Tamayo que ya había regresado de Chile, se entrevisto con el Doctor Asenjo cuando viajo a Medellín para operar a un personaje importante de la ciudad, en esta visita acordaron su entrenamiento en el

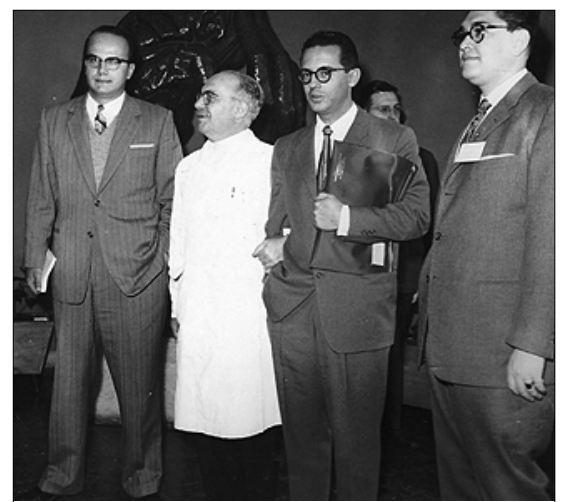

Figura 3. De izq a der Dr. C. Acebedo, Dr. Alfonso Asenjo, Dr. Ernesto Bustamante, Dr. Rodrigo Londoño. 
Instituto de Neurocirugía.

Inmediatamente después de terminar sus estudios médicos en 1947 viajo a Santiago de Chile y allí permaneció durante 2 años, fue Chile el país que le marcó su futuro médico, formándolo como neurocirujano y también motivándolo en una empresa más importante: formar un servicio de neurocirugía; en el aspecto humano allí encontró su esposa y compañera de toda la vida: Jeannie Miller con la que formo un hogar con cinco hijos: Raúl. Ana María, Roberto y María Inés, y María Victoria. Fue un residente destacado, el Doctor Asenjo resalto sus capacidades médicas en 1957, en ese año el Doctor Bustamante organizo en Medellín, el VII Congreso de la Federación Latinoamericana de Neurocirugía durante los días 8 al 11 de Julio de 1957; en el discurso dijo: "Bustamante ha sido entre los numerosos becados extranjeros que han traspasado nuestros dinteles, uno de los que dejado una trayectoria de las más fructíferas e inolvidables. Ha sido fundador de una nueva constelación neuroquirúrgica y entre sus colaboradores también estuvieron largo tiempo en Santiago otros elementos calificados, los doctores Raúl Piedrahita y Rodrigo Londoño...."10.

Regresó a Medellín en 1949 con la decisión férrea de fundar un servicio de Neurocirugía, un ejemplo que recibió de su Profesor Asenjo, con actividades científicas y asistenciales. Lo primero que hizo fue empezar clases de Neurología y Neurocirugía para los estudiantes de medicina en el mes de febrero de 1950; después el Hospital de San Vicente aprobó una sala de hospitalización con 20 camas para neurocirugía.

Se debían hacer las cirugías en un quirófano de cirugía general, sin médicos entrenados en anestesia, las cirugías se hacían bajo anestesia local y el cirujano era el responsable de recomendar los agentes anestésicos, debió entrenar el personal de enfermeras, tuvo la colaboración de su esposa Jenny como instrumentadora y poco a poco se fueron entrenando las instrumentadoras y enfermeras, se carecía de instrumentos quirúrgicos adecuados ${ }^{11}$; contó si con la colaboración del Doctor Luis Carlos Posada quien fue entrenado en Ann Arbor y había regresado a Medellín en 1948.

El servicio de Neurología y Neurocirugía se inició oficialmente el 7 de noviembre de 1950, la historia clínica nú-
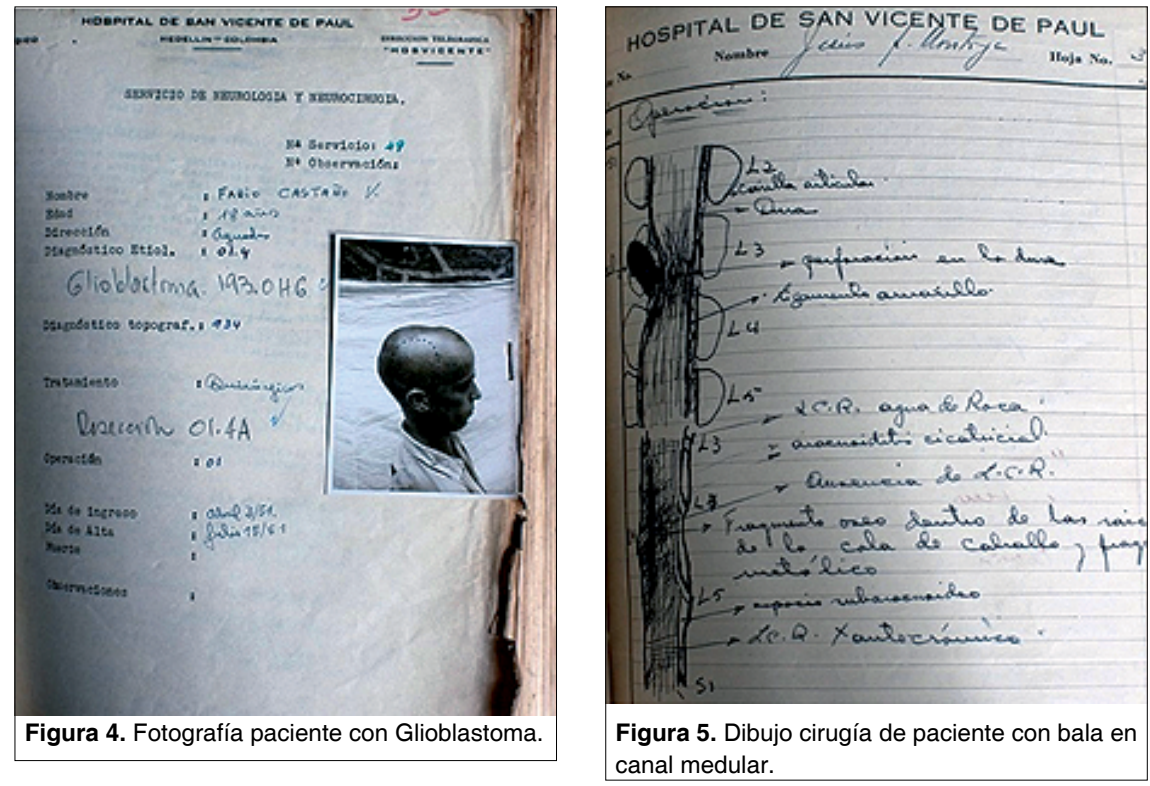

mero uno fue la del señor José Nolasco Morales quien presentaba un cuadro de compresión medular diagnosticado por mielografía, la cirugía fue hecha por los Doctores Luis Carlos Posada y Ernesto Bustamante.

Se lee en las descripciones operatorias que las cirugías se hacían con anestesia local, sedación con pentotal y atropina para disminuir la secreciones; a pesar de estas limitaciones quirúrgicas se hacían resecciones de tumores supra e infratentoriales, protuberanciales, cordotomías, discoidectomías y Psicocirugía, en las historias clínicas se pueden apreciar fotografías de los pacientes y dibujos de los hallazgos operatorios.

La técnica quirúrgica del Doctor Bustamante se caracterizó por la solemnidad y sencillez de la cirugía, la limpieza del campo quirúrgico y la simplicidad de los instrumentos que utilizo, pareciera copiada su cirugía de las ilustraciones del libro "Tratado de Técnica Neuroquirurgica" de su profesor Asenjo ${ }^{13}$.

Además del archivo de historias clínicas se hicieron los archivos de arteriografías, electroencefalografías y el archivo fotográfico con los casos más interesantes.

Aunque practicó todo tipo de cirugías, en las que más se reconoció su habilidad quirúrgica y en las que más experiencia tuvo fue en aneurismas y tumores cerebrales. Dice el Doctor Bustamante que ocluyó el primer aneurisma en la Clínica Medellín en 1949; al preguntarle sobre el tipo de anes-

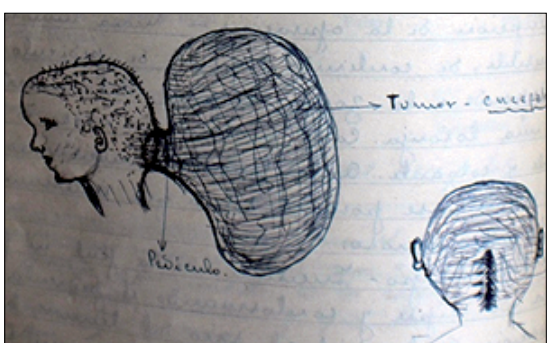

Figura 6. Dibujo de paciente con Meningocele.

tesia respondió "debió ser una buena sedación", esta fue la primera cirugía de aneurisma cerebral en Colombia, recuerda que estuvo acompañado por el Doctor Luis Carlos Posada (Neurocirujano) y Hernando Echeverri como ayudante y el instrumentador fue el Dr. Raúl Piedrahita quien años más tarde también viajó a Chile para estudiar Neurocirugía.

En los primeros años de funcionamiento del servicio algunos médicos sintieron atracción por la neurocirugía, hicieron sus tesis sobre temas neuroquirúrgicos y después se especializaron.

En esa época no estaban reglamentados los programas de residencia y quienes buscaban la especialización asistían a las actividades del servicio; se les llamaba "Internos Residentes", fue una réplica de lo que sucedía en Chile donde se hacia el entrenamiento simultáneamente en Neurología y Neurocirugía, al finalizar un período de estudios el Doctor Bustamante certificaba 


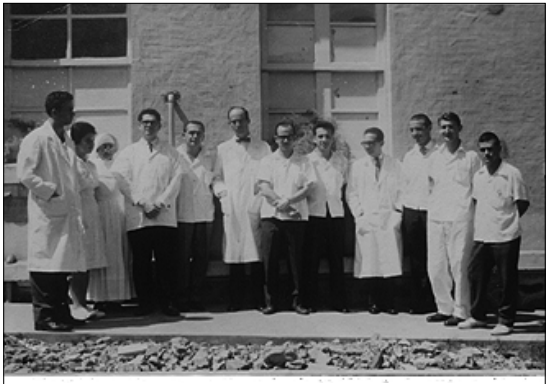

Figura 7. Grupo de profesores y residentes 1960.

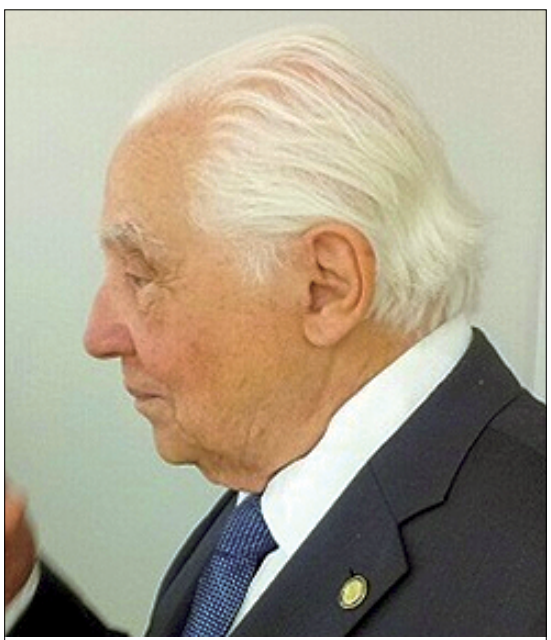

Figura 8. Dr. Ernesto Bustamante 2014.

que su discípulo era competente para desempeñarse en la especialidad. Los primeros especialistas en Neurología y Neurocirugía fueron los Doctores Rodrigo Londoño y Saúl Castaño, ellos al terminar su entrenamiento como Médico-Cirujano presentaron la tesis de grado sobre tumores cerebrales; el Doctor Rodrigo Londoño en $1954^{14}$, durante el año de 1954 fue Interno Residente en el Servicio de Neurocirugía y viajo a Chile donde fue becario del Instituto de Neurología de Montevideo y el año siguiente en el Instituto de Neurología de Chile, se desempeño como especialista en Epilepsia y Electroencefalografía. El Dr. Saúl Castaño en 1955 presento su tesis de grado sobre Meningiomas Intracraneanos ${ }^{15}$.

Durante 30 años la Neurología y Neurocirugía fueron ciencias que se estudiaron conjuntamente en el servicio pero en 1980 la Neurología fue una especialidad con un programa académico independiente.

Actualmente en el servicio de Neurocirugía y también el de Neurología se gradúan 3 residentes cada año, han sido en total más de 120 especialistas de las neurociencias que se desempeñan en toda la nación.

El Doctor Bustamante con el Dr. Luis Carlos Posada publicaron en 1952 su primer trabajo sobre aneurismas en Colombia; de 24 pacientes fueron 11 los operados y ninguno falleció ${ }^{16}$. En 1961 el Doctor Bustamante había operado 106 pacientes con aneurismas, la mortalidad fue del $24 \%$, estaba en el rango de publicaciones de la época ${ }^{17}$ en 1976 utilizó el microscopio en neurocirugía con un microscopio portátil.

Estos fueron los procedimientos quirúrgicos que mas puedo destacar porque fueron áreas en que mostró más interés quirúrgico porque también hizo todos los procedimientos quirúrgicos rutinarios de tumores, cirugía de columna y para el dolor.

Además hizo las primeras cirugías para enfermedad de Parkinson usando la técnica de Cooper, Psicocirugía, además incursionó en cirugía de epilepsia y en algunos casos realizó electrocorticografía y estimulación operatoria.

En 1960 se hizo una recopilación de las actividades del servicio durante los primeros 10 años de funcionamiento, se habían escrito 10 tesis de grado sobre aspectos neuroquirurgicos ${ }^{11}$, se habían hospitalizado 2.222 pacientes y de ellos 1.018 fueron sometidos a tratamiento quirúrgico, la mortalidad global fue del $22,3 \%$, $23 \%$ en lesiones supratentoriales y $43 \%$ en lesiones infratentoriales (en esa época se habían hecho 786 Arteriografías, 908 ence- falografías y 208 ventriculografías. El registro estadístico de 2014 es que en total fueron atendidos 7.448 pacientes en consulta externa, 5.531 del servicio de neurología (3.753 adultos y 1.778 niños), los pacientes hospitalizados por neurocirugía fueron 1.917 (1.497 adultos y 420 niños) y se realizaron en total 850 cirugías.

En toda su vida profesional el Doctor Bustamante ha estado vinculado a la vida académica ${ }^{12}$ fue Jefe del nuestro Servicio de Neurología y Neurocirugía desde su fundación hasta 1976, varias veces dijo que debíamos tener "un instituto como el de Chile". Después se traslado al Instituto Neurológico de Colombia en Bogotá, allí se desempeño como Director científico en 19781987, durante los años 1987-1992 fue Jefe de la Unidad de Neurocirugía de la Universidad Javeriana y luego director general del Instituto Neurológico de Colombia durante los años 1992-1995, aunque tiene actualmente 93 años continua vinculado a actividades académicas en la Universidad Javeriana gracias a su excelente salud física y mental.

Recuerda con mucho cariño a sus profesores, fundadores del instituto, los Neurocirujanos: Alfonso Asenjo, Héctor Valladares Arriagada, Carlos Villavicencio y Mario Contreras Villalón, además el Dr. Santiago Riesco (Otorrino) y Abraham Schweitzer (Oftalmólogo), todos ellos pioneros en su ramo. También puede recordar algunos de sus compañeros de residencia como a Alejandro Jiménez Arango, Neurocirujano

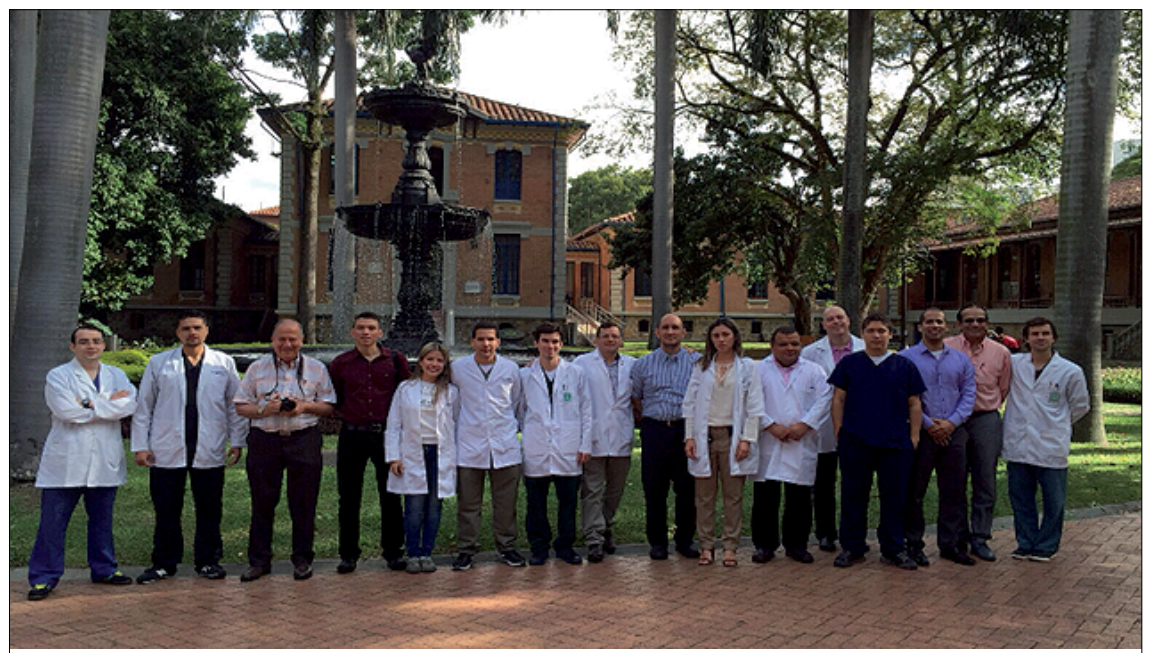

Figura 9. Grupo de Profesores y Residentes 2015. 
Colombiano que terminó un año antes, también al Doctor José Benaim quien falleció cuando dictaba una conferencia en verso sobre la historia del de la Sociedad de Neurocirugía de Buenos Aires y a otros neurocirujanos contemporáneos como los doctores: Juan Pablo Recagno Cepeda (argentino), Juan Fierro Morales, Reinaldo Poblete Grez y Paulo Niemeyer.

Entre sus profesores y amigos merece una mención especial el Doctor Héctor Valladares Arriagada (1909-2001), porque fue escogido para representarlo en la ceremonia religiosa del "matrimonio por poder", ya que el Doctor Bustamante se encontraba en Medellín, tuvo que ser su mejor amigo y que tuvieran similitud en aspectos éticos, morales y en la personalidad. Años más tarde esas cualidades fueron resaltadas en el discurso funerario porque en sus 92 años de vida, así se refería uno de sus alumnos: "Conformó ese núcleo tan homogéneo y célebre de esos médicos, (chapados a la antigua), sacrificados y esforzados, que forjaron los comienzo del Instituto de Neurocirugía, y también de esta especialidad, la Neurocirugía, en Chile". "El Dr. Valladares tuvo siempre una gran voluntad de servicio, nuestra profesión, decía, nos permite mantenemos junto a los jóvenes en la enseñanza, y junto a los humildes en la sala de los hospitales y de esta manera podemos palpar la realidad social de nuestra población". "El Dr. Valladares enseñaba con el ejemplo, poco adepto a las clases magistrales o el lucimiento con disertaciones teóricas, era elocuente sólo en su quehacer quirúrgico, en el pabellón, o en coloquios, donde entregaba sus conocimientos con mayor familiaridad y con amistad". "Por el prestigio que alcanzó en su tiempo, llegó a tener una nutrida clientela, de la cual jamás lucró, manteniendo una vida sencilla y muy austera y una disciplina casi conventual en todos los actos de su vida". "Una de sus características, tal vez la más destacada de su personalidad, fue su modestia, llevada a un nivel ejemplar, ajeno a todo boato y ostentación y desprovisto enteramente de vanidad"19.

Como lo exprese en la introducción, he descrito como nació y como se desarrolló el Servicio de Neurocirugía del Hospital San Vicente de Paul en Medellín, obra de un Neurocirujano formado en el Instituto Neurológico Alfonso Asenjo, institución que merece todo nuestro agradecimiento y admiración y con la que queremos fortalecer los lazos académicos.

Recibido: 06 de noviembre de 2015 Aceptado: 16 de noviembre de 2015

\section{Bibliografía}

1. Otalvaro D. Datos Para la Historia de la Cirugía en Antioquia, Tesis. Medellin impr. Dptal, 1892, 50 páginas. Anales de la Academia de Medicina de Medellin, vol. x: 65 - 68, 1898.

2. Bennett AH, Godlee RJ. Case of cerebral tumour. Br Med J: 988-989: 1885.

3. Arango TE. Boletín clínico, pp. 221 Boletín clínico, pág 221 Ano VIII, \# 89 Nro 5, Sept. 1942.

4. Gómez J. Bustamante E. Gil JG. Espongioblastoma Multiforme Precoronal. Boletín Clínico, Vol., 09, Núm. 02 Oct. Pp. 55-58, 1947.

5. Bustamante E. Yodoventriculografía, Diagnóstico de un tumor de la Pineal, Boletín Clínico 9 (2): 67-73, 1946.

6. Bustamante E. La Yodoventriculografía en el diagnóstico de tumores intracraneanos, Tesis 1947.

7. Sion A. Arteriografías periféricas y angiografías cerebrales, Tesis, U. De A. 1948.

8. Arango O, Arango O. Estudio Clínico Radiológico De La Hernia De Disco Intervertebral 9 \# 3:81-91, 1947.

9. Llano EG. Ciática y Lumbociática por lesión de disco intervertebral, Tesis, 1947.

10. Asenjo A. Prólogo, Neurocirugía (Chile) vol XV 3 1- 2, pp. 7-8, 1957.

11. Bustamante E. Reseña Histórica del Servicio de Neurología y Neurocirugía del Hospital Universitario San Vicente de Paul Antioquia médica Vol 10 Núm. 9 Oct. pp 464-468, 1960.

12. Bustamante E. 25 Años de un Neurocirujano en Colombia. Antioquia Médica 25:(4-5) 319-1:324,1975.

13. Asenjo Alfonso. Tratado de Técnica Neuroquirúrgica, Editorial Inter-médica, Buenos Aires, 1959.

14. Londoño R. Tumores Intracraneanos, Tesis, 1954.

15. Castaño Saúl. Meningiomas Intracranenos, Tesis de Grado, 1955.

16. Bustamante E, Posada, LC, Carmona R. Hemorragias subaracnoideas y aneurismas intracraneanos, Antioquia Médica, Vol 2, \# 5 , pág 341-348, 1952.

17. Mejia A. Francisco Vélez AID. Aneurismas arteriales intracraneanos, estudio de 106 pacientes, Tesis 1961.

18. Bustamante E. Estadística del Servicio de Neurología y Neurocirugía 1950-1960, Antioquia Médica, Vol. 10, \# 9, pp. 469-471, 1960.

19. Díaz PG. Palabras en los funerales del Dr. Héctor Valladares Arriagada Rev. Chil. Neuro-Psiquiatr. Vol .39 n.2 Santiago abr. 2001.

Correspondencia a:

Luis Carlos Cadavid

stxcadavid@gmail.com 